\title{
INDEX OF MEDICAL CONDITIONS
}

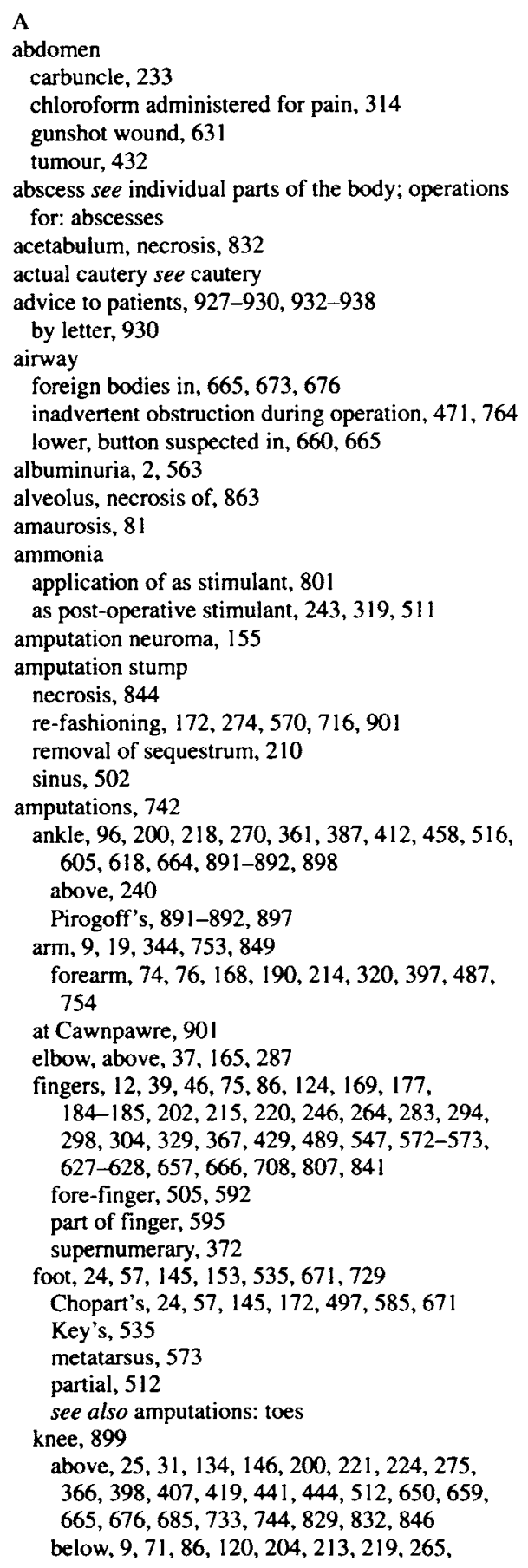

amputations, knee, below (cont'd):

$311-312,314,361,487,552,575,657,685$, $700,728,772,790$

through, 525

leg, $19,46,69,348,851,913$

in Crimea, 689, 716

penis, $184,254,270,277,419$

performed without anaesthesia, $46,779,851$

Syme's, 96, 618

thigh, 32, 58, 74, 275, 294, 341, 378, 455, 525, $566-567,572,578,589,612,644,762,809$

high, 547

stump of, 468

thumbs, $192,251,307,350,387,389$

supernumerary, $60,219,502,556$

toes, 19, 26, 38, 66, 106, 157, 188, 206, 212, 232, $240,260,274,303,385,508,676,682,740$,

$758,809,832$

great, 577

wrist, 86, 849, 896

Amussat's operation, 280, 794

amylene

deaths from, 782-784, 845

from Dr. Debout, 779

runs out during anaesthesia, $759,763,833$

Snow's first mention of in Case Books, 736

anaemia see co-existent diseases

anaesthesia

advice against use of, 578

awareness during, 551, 776, 874, 895

feigned by patient, 825

deaths

from amylene, $782-784,845$

under chloroform, 408-409

operations without, $46,779,851$

see also amylene; chloroform; ether; induction of anaesthesia

analgesia, post-operative, 47, 78, 732

aneurism see operations for: aneurisms

ankle

abscess near, 669

bone, necrosis of, $71,504,765,870$

sinus near, 525

tenotomy, 219,915

see also amputations; tarsus

ankylosis of knee, 567

antrostomy, maxillary, 197

anuria, 133

anus

abscess, near, 672

artificial, 280,794

post-operative incompetence of, 637

tumours, 276, 349

see also Amussat's operation; operations for: anal, anus, fissures, fistula, perianal; sphincterotomy 


\section{Index of Medical Conditions}

arcus senilis, $243,365,371,528,584,588,592$, $596,611,641,646,777,898$

arm

cicatrix, 610

burn, 755

forearm

bone, necrosis of, 614

dislocation of, 880

foreign body in, 307

fracture, 612

manipulation of, 843,880

naevus, 640

naevus, 638

necrosis of bone in, 623

neuralgia of, 574

neurectomy for pain in, 574,626

tumours, $19,345,379,440,649,706$

see also amputations; humerus; radius; ulna; wrist arrhythmia during lithotrity, 596, 598

arrow root, 528

artery

axillary, ligation of, 192

common carotid

aneurism of, 398

ligation of, $305,398,891$

facial, division of, 660

femoral

aneurism of, $67,204,222,351,677,725,785$

legation of, $257-258,351,795$

ophthalmic, aneurism of, 891

popliteal, aneurism of, $67,204,351,677,785$

radial, ligation of, $77,307,847$

temporal, aneurism of, 269

ulnar, ligation of, 847

artificial ventilation, Marshall Hall's method, 783

assafoetida, 541-542

asthma

chloroform administered for, 239, 391

co-existent disease, 251

auscultation of heart by Snow, 851

awakening during operation, partial, 22, 802

axilla

abscess, 683

burn contractures near, 633

naevi, 716,726

see also operations for: tumours

axillary glands, 239

Aztec, child who looked like an, 537

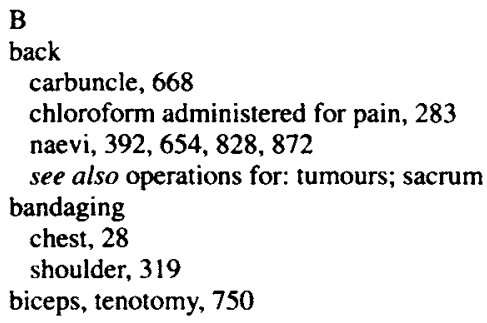

biceps, tenotomy, 750

bistoury, use of, 353

bite of a horse, 726

bladder

examination, $291,498,712$

for stone, 604

foreign body in, 54

irrigation of, 320

sequestrum in, 192

sounding of, $214,216,224,256,270,272,285$,

$289,327,408,423,442,571,594,597$,

$617-618,640,644,648,654,658,694,701$,

$730,732,784,839,846$

see also lithotomy; lithotrity

blindness

co-existent disease, 59

see also amaurosis

blood, intra-operative inhalation of, 184

bone diseases

ankle, necrosis near, 534

arm, necrosis, 623

carpal and metacarpal, necrosis, 38

finger, necrosis, 482

foot, necrosis, $257,497,703,805$

hand, 855

necrosis, 403,633

syphilitic disease, 598

wrist, necrosis, 497

see also operations for: necrosis

bouginage, 30I, 303, 306, 309, 353

brain disease, co-existent disease, 903

brandy, as post-operative stimulant, 852,884

breast

naevi, $141,320,340,606,632,653,668$

removal of, 12, 19, 82, 108-109, 111, 113, 122 , $142,161-162,177,184,191,226,237,244$, $263,266,269,273,331,339,656,864,895$ sinuses, 153

breast tumours

Dr. Fell's method of treating, 875

removal of, $19,27,31,35,37,41,62,70,80-81$, $98,148-150,157,169,173,177,197,204,206$, $211,217,219,222,233-234,240,245-246$, $254,261-262,265,275,278-279,285,291$, $293,296,308,312,320,323,326,330-331$, $336,339,351,355,357,365,371,374-375$, $379,382,384,386,389,399,401-402,412$, $416,422,424,432-433,436-438,440-441$, $443,446,450-452,457,462,464,476,484$, $486-487,491,498,501,503-504,507,513$, $525,530,535,548,566,572,575-576$, $579-580,584,592,602,607,610-611,620$, $624,626,631,634,635,656-658,661-662$, $666-667,670,673,677-678,680,686,688$, $689,691,693-695,704,710-711,713,716$, $720,726,731-732,742,750,760,766$, $768-769,775-776,794,827,829,832,840$, $842-843,845-846,848,850,852,855,859$, $861,868-870,872-873,875,886-887,890$, $899,913-915$ 


\section{Index of Medical Conditions}

breast tumours, removal of (cont'd):

in a male, 168,191

weighing $181 / 2 \mathrm{lbs}, 469$

bronchitis

co-existent disease, 354,756

chronic, 400, 619, 656, 802

bullet wounds see operations for: bullet wounds, gunshot wounds

bums see operations for: burn contractures, cicatrices,

bursa patellae, $257,279,422,723,806,892$

bursae see operations for: bursae

buttocks

sinuses, 850

tumours, 703

see also nates

C

calculi

intestinal, 524

see also operations for: stone; prostatic calculi

calomel, 541, 586, 616

cancer

internal, 695

see also operations for: cancer

cancrum oris, 306

capsulectomy of lens, 826

carbuncle, 845

see also operations for: carbuncles

cardiac disease see co-existent diseases; heart

carpal bones

excision of, 318

and metacarpal bones, necrosis of, 38

carpus see operations for: necrosis; wrist

catalepsy, 836

cataract see operations for: cataract

catheterisation, $60,89,94,116,119,170,183,206$,

$209,269,301,311,321,364-367,416,459,462$,

$464,470,472,504,679,704,751,758,764,900$.

902

pre-anaesthetic, 754

caustic, application of, 14, 33, 113, 123, 148, 170 , 288-289, 297, 299, 313, 373, 392, 449, 456,

$575-576$

cautery

actual, $35,233,583,650,728,803,855-856,878$, $888,897,908$

electro-, 803-804

cervix, tumours, 35

cheek

aperture in, 750

bone, tumour, 165

bullet wound, 603

cancer, 603

cicatrix, 244

disease of, 461

fissure, 631

naevi, $147,236,262,361,375,432,434,544$,

682,707 cheek (cont'd):

opening in, 637

sinuses, 836

see also operations for: tumours

chemosis, 695

chest

bandaging, 28

cyst, 234

disease in a baby, 5

naevi, 539,671,674

tumours, 207, 281, 394

chimney-sweeps' cancer, 507,648

chin

naevus, 750

tumour near, 753

chloroform

advice against the use of, $56,199,284,406,595$, 722,866

caution advised by physician, 490

contaminated, 593

local application

for pain, 46, 63, 195-196

for post-operative pain, $92,147,180,281$

possible sequelae of, 358

runs out during administration, 55

Snow gives for amusement, 163

see also inhaler; sponge; towel; valves

chloroform administered

by person other than Snow, 481, 517, 542, 558 $563,599,622,626,645-646,687-688,695$, $702,732,751,786,856,876,894$

surgery not performed, 481

to detect feigning, 335, 343, 451-452

chloroform administered for

abdominal pain, 314

application of splints, 395

asthma, 239, 391

back pain, 283

cholera, 125, 133

delirium tremens, 341

dressing a diseased knee, 346

facial pain, 195-196, 204-205

heart disease, 447

hyperemesis gravidarum, 381

indication not stated, 17

insomnia, 400

laryngeal obstruction, 328

laryngismus stridulus, $428,433-436,438,454$

515

mania, $322,403,853$

neuralgia, 40,730

post-operative analgesia, $47,78,732$

post-operative pain, 147, 180, 281

tetanus, 64-65, 506, 892

see also examinations

chlorosis, co-existent disease, 762

cholera

inhalation of chloroform for, 125,133

inhalation of Dutch Liquid for, 125 


\section{Index of Medical Conditions}

cholesterine, 197

Chopart's amputation, $24,57,145,172,585,671$

chorea

co-existent disease, 878

as a side effect of morphia, 34

cicatrices see operations for: cicatrices

circumcision, $33,61,79,167,188,203,220,248$,

$254,283,311,368,405,444,621,624,626,628$,

$639,668,690,707,787,793,798,803,844,848$, 858,911

clavicle

cyst near, 94

fractured, 223, 929

naevus near, 758

necrosis of, $349,589,658$

tumours near, 97,555

cleft palate

co-existent disease, 473

see also operations for: cleft palate

club foot see operations for: club foot

co-existent diseases

anaemia, $541,553,555,577,648,695,723,738$,

$757,810,841,846,884,920$

asthma, 251

blindness, 59

brain disease, 903

bronchitis, 354, 756

chronic, $400,619,656,802$

cardiac, $21,44,56,141,447,474,532,576,578$, $584,606,648,651,662,667,723,792,802$.

$859,866,898,910$

cavitation of lungs, 50 l

chlorosis, 762

chorea, 878

cleft palate, 473

congenital deafness, 290

conjunctivitis, 838

cor pulmonale, 400

deaf mutism, 72

deafness, 59

depression during pregnancy, 509

dwarfism, 752

dyspnoea, 903

eczema, 838

emaciation, 477,512

emphysema, 802

epilepsy, 199, 755, 832, 896-897

generalised oedema, 186

hemiplegia, 9, 212, 335, 562, 646, 651, 730

insanity, 36

nervous seizures, 859

obesity, $142,150,154,251,468,490-491,495$, $536,556,609,657,663,698,733,752,763$. $841-842,845,866$

ovarian tumour with debility, 761

palpitations, 65

phthisis, 530,534

polycythaemia, 52

renal disease, 903

rheumatic heart disease, 474 co-existent diseases (cont'd):

rickets, 471

spinal disorder, 900

tetanus, 64-65

tuberculosis, 706, 878

cod liver oil, medication with, 501

cold, local application, harmful effect of, 570

collar-bone see clavicle

collodion, 86-87

conjunctiva see eyes

conjunctivitis, co-existent disease, 838

consultations with colleagues, $2,18,68,71,101$, $107,122,160,928,932$

convulsions

chloroform inhalation for, 541,774

death of a child after, 543

during anaesthesia, 32

cor pulmonale, co-existent disease, 400

cornea see eyes

craniectomy, 45

cricket ball

bone necrosis due to blow from, 601

injury from, 881

croup, chloroform inhalation for, 614,619 , 622-623, 674

cupping, 174

pre-operative, 586

cysts see operations for: cysts

D

deaf mutism, co-existent disease, 72

deafness, congenital, co-existent disease, 290

deaths

of child, intra-partum, 94

during chloroform anaesthesia (Mauritius), 196

during Snow's amylene anaesthesia, 782-784, 845

during Snow's chloroform anaesthesia, 408-409

from hydrocephalus, 774

from meningitis, 616

from overdose of Dover's Powder, 252

from peritonitis, 631

from typhoid fever, 868

neonatal, 49,98

post-epileptic, 563

post-operative, $35,41,45,47,155,162,182-183$, $200,217-218,251,273,321,332,334,382$,

$390,402,444,448,460,469,478,503,512$,

$516,524,545,547,550,560,570,572-573$,

$579,590-592,625,631,634,667,695,699$.

$714-715,731,735,742,785,794,828,844$,

$850,889,913$

postmortem findings in, 667

post-partum, 332, 360, 585

delirium tremens, 562

inhalation of chloroform for, 140, 193-194, 341

deliveries, 4, 23, 28, 30-31, 39, 42, 48, 51, 54, 68,

$75,87,98,116,124,129,136,139,144,151$,

$160,181,184-187,207,219,227-228,232,250$,

$265,293,313,325,346,366,419,427,439,455$, 


\section{Index of Medical Conditions}

deliveries (cont'd):

926, 929, 931-932, 937

forceps, 94, 107, 702, 713, 721, 761, 778, 906

premature, 926

see also labour; pregnancy

dental extractions, $13,21,34,41,43,49-51,56-57$. $62,65,67,72,84,89,101-103,110,114-115$, $123-124,127-128,131,156,176,188-189,198$, $202,208,214,222,228,230,233,242-244,247$, $250,255,259,277-280,286,289-293,295,306$, $314,324,327,330-331,333,338-339,342-343$, $345,349,352-353,356-358,362-363,367,369$, $372-376,379-381,383-384,386,388,391-394$, $396,398-399,402-404,407-408,410,412-413$, $415,417-418,420-421,423-424,426-430,432$, $436,439,441-442,446-447,450-451,453-454$, $456,459-463,468-469,472-476,478-480$, $482-488,490-491,493-494,496,499,503-505$, $511-516,518-519,521,523-524,526-528$, $531-532,534-541,543-551,553-554,556-560$, $562,564,566-568,571-575,577-578,581-584$, $587-588,593-595,598-599,601,603-610,614$, $617,620-649,651-652,656-659,662-664,666$, $669-677,679-685,687-690,692-693,695-698$, $700,702-703,705-713,715-719,721-723$, $725-731,733-735,737-742,744-750,753,756$, 758-762, 764-773, 775-776, 779, 784, 788-791, 794-795, 797-805, 807-811, 825, 829-831, 833, $835,837-839,841-844,846-847,849-850$, $852-867,869,871-882,884-890,894-897$, $899-904,906-919$

abandoned, 751

performed by Snow, 13

dental filling with gold, 643

dental neurectomy, $90,121,388,459,483,485$, $495,553,575,633,693,700,743,758,786,874$, 879,880

dental prop, use of a cork as, 498

depression during pregnancy, co-existent disease, 509

diagrams drawn by Snow, 45, 95, 618

dilator used to open jaws, 490

dislocations see operations for: dislocation

Dover's Powder, death from overdose, 252

Dutch Liquid, 93, 107, 117, 125, 136

dwarfism, 752

dysentery, 225

dysmenorrhoea, 41, 163, 197

dyspareunia, 692

dyspnoea, co-existent disease, 903

E

ears

cancer, 887

foreign bodies in, 103, 799

lobe, accessory, 565

syringing of, 164

tumours, 19,63

behind, 584 ears (cont'd):

see also operations for: polyps

écraseur, use of, 734, 757

eczema, co-existent disease, 838

elbow

abscess, 750

burn contracture, 633

burns at joint, 66

disease of, 409,411

dislocations of, $62,344,396,644,652$

fracture, 337

manipulation of, $655,661,750,780,808$, $847-848,864$

necrosis of, 148,190

resection of, 137

tumours, 312

near, 700

see also amputations; excisions

electro-cautery, 803-804

elephantiasis see prepuce

emaciation, co-existent disease, 477,512

emphysema, co-existent disease, 802

enchondroma see operations for: enchondroma

epididymis see operations for: tumours

epilepsy, 322,343

chloroform inhalation for, $105-106,215$

co-existent disease, 199, 755, 832, 896-897

consultation by Snow about a case of, 562

death following fits, 563

inhalation of valerianate of oxide of ethyle for, 129

ergot, 553, 642, 786

erysipelas, 714, 935

post-operative, fatal, 572-573

ether anaesthesia, alarming reaction during, 207

examinations

airway, foreign body in, 665

bladder for stone, 604

bone of toe, 643

foot, 888

hip, 763, 888

knee, 705

larynx and bronchi for a foreign body, 660

neck, deformed, 912

patient at Royal Oak Benefit Society, 938

rectum, 443, 679, 734-735, 757, 900

malignancy, inoperable, 653

scapula, deformity of, 774

under chloroform prior to surgery, 828

vagina, malformed, 799

excisions

carpal bones, 318

elbow, $181,387,412,421,534-535,545,552$, $560,596,620,653,667,701,724,728,801$, 832,837

eyeball, $104,156,594,674,678,688,695,698$, 703-704, 708, 725, 731, 746, 751, 753, 757, $762,764,796,837,841,848,850,862,865$, $882,889,896,905,912-914,918,920$

for post-operative complication, 678 


\section{Index of Medical Conditions}

excisions (cont'd):

femur, head of, 132

keloids, 913

knee, $218,416,448,575,582,594,674,680,691$, $697,715,725,729,795,806,809,828,841$, 865,914

labia pudendi, 113

tibia, 218

toe-nail, 346

excitement

during induction, $12,33,57,172,200,218,295$, $326,353,381,401,538$

post-anaesthetic, $468,531,744,801$

exostosis see operations for: exostosis

eyeball see excisions

eyebrow

cyst, operation for, 333

naevus, 379

see also operations for: tumours

eyelids

adhesion, 293

cancer, 624, 909

near, 789

cancroid disease, 508

cicatrix, 344

deformity, 698

disease of, 478, 771

refashioning of, 655

fashioning of upper, 618

growths, 871

naevi, $411,427,470,571,573,657,700,725,878$

see also operations for: tumours

eyes

adhesion, 288

capsulectomy of lens, 826

conjunctiva

adhesion, 260

growths, 673

inflamed, 752

cornea, foreign bodies in, $704,707,737$

corneal deposit, 611,768

cyst, sebaceous, near, 203

disease, $384,388,477,760,807$

foreign body in, 339, 421,770

gun accident, operation for, 767

inflamed, ether inhalation for, 91

inflammation, 916

iridectomy, 903, 907, 915, 918,920

partial, 876, 883, 909

iris, protrusion of, 712

melanorna, 47 !

melanosis, 751

naevus near, 630

operation, nature of omitted by Snow, 759

ophthalmia

purulent, 694

sympathetic, 731

orbit

growth, 871

injection of sclerosant, 725 eyes, orbit (cont'd):

swelling involving, 557

tumours, $60,118,305,617,634,653,680$

near, 33,558

percussion cap in, 770

pupil, artificial, $406,412,552,555,754,788$,

$862,864,906$

traumatized, 681

tumours, 95, 522

near, 35, 641, 677

see also conjunctivitis; operations for: cataract, strabismus

F

face

cicatrices, 836,859

and on neck, 882

disease of, 392

fissure, 565

naevi, 184, 270, 389, 714

nitric acid applied to, 470,538

sinus, 863

see also cheek; chin; facial pain; facial palsy;

forehead; jaws; lips; nose; operations for:

tumours

face-pieces see inhaler

facial pain, $26,90,640$

chloroform administered for, 195-196, 204-205

neurectomy for, 488,640

facial palsy, post-operative, 709

faeces, dis-impaction of, 803

fasciotomy, plantar, 232, 240, 796-797

feigning, patients suspected of, $335,343,451-452$

femoral artery, ligation of, 257-258

femoral strangulated hernias, 334, 554, 688

femur

bullet wound, 903

disease of, 606

dislocation of, 867

distortion of, 488

enchondroma, 791

excision of head of, 132

fractured, 751

necrosis of head of, 202

tumour, 120

see also thigh; trephining

fibula

necrosis of, 904

and tibia, fractured, 203, 872

fingers

burn cicatrices, 468

bursa, operation for, 375

enchondroma, 604

exostosis, 719

fusion of, 601

manipulation, 890

necrosis of bone, 482,496

necrosis of, 378,426

supernumerary, 372 


\section{Index of Medical Conditions}

fingers (cont'd):

tenotomy, 738

tumour, 599

webbed, 776, 780, 804

see also amputations; hand; nails; thumb

firing iron

application of, 233, 855

to knee, 612

fissures see operations for: fissures

fistula lachrymalis, 728

fistulae see operations for: fistulae

flank

tumours, 238, 267

see also side

flexion deformity of knee, 343

foetal head

moulding of, 30,553

perforation of, 117

foot

bandaging of, 12

examination of, 888

foreign bodies in, 906,917

gangrene, 675

lancing of, 769

necrosis of bone in, 257, 497, 703, 805

tumours, 25, 737

see also matatarsus; metatarsal bone; operations for: club foot; tenotomy; toes

forceps

application of obstetric, 647

see also deliveries

forearm see arm

forehead see operations for: naevi, tumours

foreign bodies

in airway, examination for, 665

see also operations for: foreign bodies

fractures see operations for: fractures

fungus haematodes, 592, 605

\section{G}

ganglion, 367

gangrene, of foot, 675

gonorrhoea, acute, 311

groin

abscess, 494

melanoma, 761, 863

sinuses, 729

see also operations for: tumours

guano, scrotal cancer in a worker with, 648

gum

boil, 13,640

lancing of, 13, 706

gunshot wounds see operations for: bullet wounds, gunshot wounds

\section{$\mathrm{H}$}

haemoptysis, $1-2,42,132$

haemorrhage, $27,59,79,84,96,145,155,158$. haemorrhage (cont'd):

$177,190,216,218,222,248,259-261,263,270$, $309,312,317-318,322,345,379,390,415,489$,

$528,535,545,547,549,561,577,590,607-608$,

$613,620,627,642,695,699,709,711,720,722$.

$766,794,829,834,841,846-847,864,884,898$,

913,915

post-partum, 49,55

secondary, 25, 321, 665

haemorrhoids

nitric acid applied to, 524, 650, 797

see also operations for: haemorrhoids

hand

abscess, 405, 599

burn contractures, 29l, 854

cancer, 711

cicatrix, 70

naevus, 75

tenotomy, 460

tumours, 148, 371, 378

see also fingers; metacarpal; metacarpus

handkerchief

chloroform on, $548,595,613,616,687,735,786$

chloroform and spirit on, for typhoid fever, 868

hare-lip see operations for: hare-lip

head

foetal

moulding of, 30,553

perforation of, 117

injury, 44

naevi, 200,459

occiput, naevus, 334

tumour, 116

see also scalp; skull

heart

auscultation by Snow, 851

disease, chloroform administered for, 447

irregular, 536, 557, 629, 711, 850

see also arrhythmia; co-existent diseases; rheumatic heart disease

hemiplegia, co-existent disease, 9, 212, 335, 562 , $646,651,730$

hernias, 375,875

incarcerated omental, 382

re-exploration, 375

taxis for, 510

tumour, 510

see also operations for: strangulated hernias

Heurteloup's table, 273

hip

abscess near, $567,602,827$

disease of, 409,426

dislocations of, 109, 221, 718

examination of, 763,888

manipulation of, $494,751,768-769,780,807$, 867

tumour, 488

see also acetabulum 


\section{Index of Medical Conditions}

\author{
hooping cough \\ chloroform for, 517-518 \\ death from, 518 \\ humerus \\ bullet wound, 680 \\ dislocation of, $183,512,611,743,751$ \\ exostosis, 716, 764 \\ fracture, non-union of, 29 \\ see also arm; operations for: necrosis \\ hydrocele, 147, 199, 292, 900 \\ iodine injection of, 900 \\ hydrocephalus, 774 \\ hyperemesis gravidarum, chloroform administered \\ for, 381 \\ hyperventilation, 617 \\ during inhalation of chloroform, 496, 501 \\ post-anaesthetic, 528 \\ hypochondrium, abscess, 670 \\ hysteria, 532, 775, 840, 860 \\ during induction, $735,752,835$ \\ post-operative, $44,58,121,128,153,172,191$, \\ $472,579,587,637,639,641,741,748,755$, \\ $758,772,801,810,827,836,838,842,855$. \\ 860,867 \\ post-partum, 527 \\ hysterical behaviour, 11
}

I

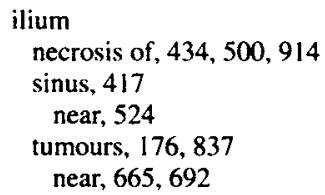

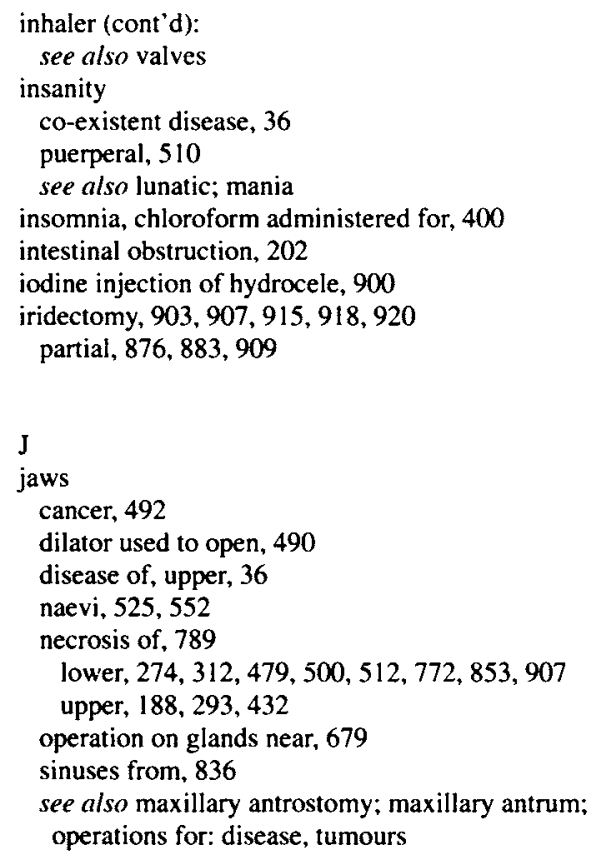

L

labia pudendi

cancer, 525

division of congenital fold, 355

excision of, 113

tumour of condylomata of, 113 


\section{Index of Medical Conditions}

labia pudendi (cont'd):

see also operations for: tumours, warts

labour

amylene in, 746, 778, 792, 796, 800, 806, 834

chlorated muriatic ether in, 332

chloroform in, $23,29,39,48,55,87,94,117$, $138,151,161,207,209,227,263,276,284$, $296,353,359-360,386,388,448-449$, $454-457,461,477,488,492,499,501,505$, $509,526,548,552,558,563,573,584,600$, $609,612-613,622,625,642,646,649,652$, $657,664,678,687-688,695,702-703,713$, $717,720-721,723-724,761,785-786,804$, $834,856-857,865,873,876-877,886,905$

Dutch liquid in, 93, 107, 117, 136

induction, of premature, 649

lingering, 717, 856

premature, 563

Snow gives chloroform for Queen Victoria's, 448-449, 785-786

version under chloroform, 602,805

see also deliveries; forceps; placenta;

presentation; version

lancing of gum, 13,706

laparotomy, 181-182

for pelvic tumour, 157

laryngeal obstruction, chloroform inhalation in, 328

laryngismus stridulus, 428, 433-436, 438 454, 515

laryngotomy, 660, 672

chloroform via a, 665

larynx

and bronchi, examination, 660

tumour, 617

see also laryngotomy

lead, suture formed of, 804

leeches, 533, 541, 555, 936

legs

abscesses, 514, 733

deformity of, 257

manipulation of, 840

sinuses, 743

tumours, 74, 327, 453, 704

see also amputations; ankle; thigh; tibia

lemon juice and diluted sulphuric acid as a tonic, 934

'leucophlegmatic', 609

lever, mouth opened with a, 831

ligation of

axillary artery, 192

common carotid artery, 305, 398

femoral artery, 257-258, 351, 795

radial artery, $77,307,847$

spermatic veins, 771

ulnar artery, 847

lips

burn, 826

cicatrix, 589

deformities, 669,733

lower, 637

division of, 724 lips (cont'd):

double, 508

fissure, on upper, 531

loss of, operation for, 732

naevi on upper, 504, 825

and nose, operation, 697

sloughing of lower, 708

see also operations for: cancer, hare-lip, naevi, tumours

lithotomy, 27, 54, 59, 76, 89, 92, 95-96, 113, 137 , $150-151,154,169,172,182,191,217,236-237$, $251,255,260,262,276,287,293,305,325,327$. $329,334,357,372,382,390,407,426,431,455$, $469,477,481,540,552,560,572,581,585$, 587-588, 592, 599, 619, 624, 630, 635, 644, 646, $649,656,662,667-668,671,677,689,699,721$, $733,742,745,754,766,772,785,788,826,836$, $859,871,895,901-902,910,912$

complicated by recto-vesical fistula, 277

see also bladder; lithotrity; operations for: stone lithotrity, 47, 126-127, 186-187, 208, 211-212, $214,220,222,229-230,236-237,242,249,251$, $255-256,261,263,268,271-273,276-278,282$, $286,288-289,291,294-295,302-303,306-307$, $314,318,320-321,323,340,344-345,347,350$, $365-367,369-370,372-374,386,390,398-400$, $403,406,408-409,413,415-416,418,430,432$, $440,443-445,450,457,462,470,472,475,477$, $479-480,482-484,486,492,495,497-498,500$, $503,510,514-516,568,570,586,588-589,591$, $592,596-597,606,609,612-613,617,637,639$, $648,650-651,653,660,691,699,718,721-722$, $724-726,728,730,739,754,776,779,781,787$, $840-841,844,846,860,866,903$

for retained bougie, 702

see also arrhythmia; bladder; lithotomy; operations for: stone

litmus paper, test of expired air with, 563

lockjaw

chloroform administration for, 506

death from, 506

loin, tumour, 402

lunatic, a, 739

lungs

cavitation of, co-existent disease, 501

see also bronchitis; emphysema

M

mandible see jaws, lower

mania, chloroform administered for, $322,403,853$ manipulation

of elbow, $655,661,750,780,808,847-848,864$

of finger, 890

of forearm, 843,880

of hip, $494,751,768-769,780,807,867$

of knee, $642,722,810,831,835,843,855,885$, $887-889,891$

of leg, 840

of neck, 591 


\section{Index of Medical Conditions}

manipulation (cont'd):

of shoulder, $780,799,827$

of thigh, 591

masturbation, 315

maxilla see jaws, upper

maxillary antrostomy, 197

maxillary antrum, tumours, 210,417

meatus urinarium, tumours, 508

Medical Times and Gazette, case reports in, 409, 742,845

melanoma of chest wall, 248

melanosis of the eye, 751

melanotic tumours, 248, 260, 365

meningitis, chloroform inhalation for, 615-616

metacarpal and carpal bones, necrosis of, 38

metacarpus, necrosis of, $516,683,917$

metatarsal bone, necrosis of, 673

metatarsus

amputation, 573

see also operations for: necrosis

microstomia, 537

morphia, 853

chorea as a side effect, 34

mouth

aperture in roof of, 517

bullet wound, 603

burn contracture, 634

gum, cancer, 484

gum boil, 13, 640

opened with a lever, 831

tumours, 233, 785

see also cleft palate; tongue

muscles

gastrocnemius, tumour beneath, 516

recti, divarication, 722

sterno-mastoid

division of, $539,556,768,826$

tumour near, 839

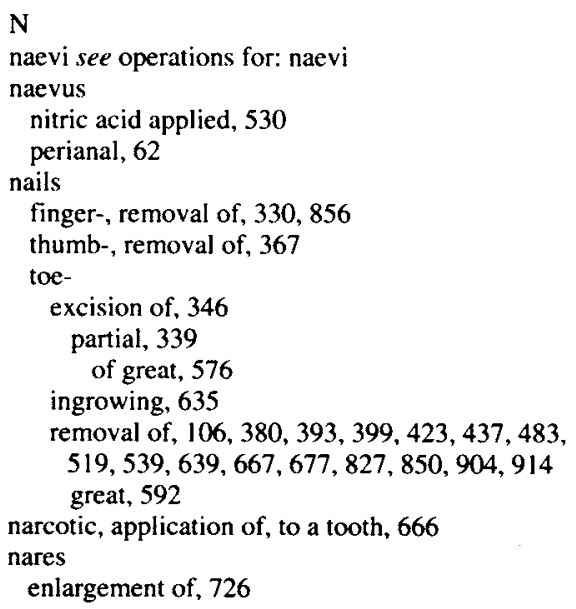

nares (cont' $d$ ):

see also nose; operations for: nasal disorders, polyps

nates

tumours, 203, 379, 569, 718-719

see also buttocks

neck

carbuncles, $628,739,742,910$

cicatrices, 488,859

and face, cicatrix, 882

cyst, 239

deformed, examination of, 912

manipulation of, 591

wry, 188

see also operations for: naevi, tumours; throat

necrosis see operations for: necrosis

neonatal

asphyxia, 30

deaths, $49,98,130,152$

resuscitation, $30,130,152,650,702$

nerves

median, tumour, 902

posterior tibial, tumour, 630

see also neurectomy; neuroma

nervous seizures, co-existent disease, 859

nettle rash, 91

neuralgia

chloroform for dental, 40

with hemiplegia, 730

post-partum, 499

see also operations for: neuralgia

neurectomy

for arm pain, 574,626

digital, 594

for facial pain, 488,640

infra-orbital nerve, $26,148,219,285,406,417$

median nerve, 172

mental nerve, $145,285,401,406,417,420,464$

of thumb, 389

trigeminal nerve, $166,187,508,523,589,854$

see also dental neurectomy

neuroma

amputation, 155

sciatic nerve, 468

nitric acid, application of to, 856

child, 533

face, 470,538

haemorrhoids, $524,650,797$

naevus in a child, 530

prepuce, 514,629

rectal fissure, 650

thumb, 765

tooth, decayed, 578

nitrous oxide, inhalation of many years previously, 564

nose

cancer, operations for, 19,726

deformity, 733

fashioning of new, 733

fashioning of new septum of, 567,588 


\section{Index of Medical Conditions}

nose (cont' $d$ ):

loss of, 726

naevi, 361,389

plastic repair of, $177,268,458,697$

refashioning of new, 740

sinus of bridge of, 519

syphilis, 912

vaccination on, 458

see also nares; operations for: nasal disorders,

polyps, tumours

nose bleed, 934

nurse, a mistake made by, 514

nystagmus, 532

O

obesity see co-existent diseases

obstructions, airway, inadvertent during operation, 471,764

oedema, co-existent disease, 186

onychia maligna, 202

operations

abandoned, $117,119,141,158,220,281,404$

awakening during, partial, 22, 802

combined, surgeon and dentist, 603

Palorcotian, 707

performed without anaesthesia, 46, 212, 340, 520 .

596-597, 697

postponed by surgeon during anaesthesia, 839

unspecified, 411

see also amputations; breast, removal of; breast

tumours; capsulectomy; circumcision;

excisions; iridectomy; laparotomy;

laryngotomy; lithotomy; lithotrity; neurectomy;

orchidectomy; sphincterotomy; tenotomy;

tracheostomy; trephining

operations for

abscesses

ankle, near, 669

anus, near, 672

axilla, 683

breast, 201, 741, 882

calf, 733

elbow, 750

groin, 494

hand, 405, 599

hip, near, 567, 602, 827

hypochondrium, 670

knee, 774, 796

leg, 514

pelvic, 828

perianal, $502,554,564-565$

perineal, 52, 54, 636, 741, 759

rectum, 651,881

shoulder, near, 625

thigh, $164,399,514,668,685$

thumb, 392

toe, 767

accessory ear lobe, 565 operations for (cont'd):

adhesions

conjunctival, 260

eye, 288

eyelid, 293

penile, 147

prepuce. 689

vagina, 429

amputation neuroma, 155

anal

excrescence, 43

polyps, 479

prolapse, $8,35,126,294,299,311.313,338$,

$341,382,412,446,462,860$

skin folds, 539

tags, $1,82,231,237,307,309,350,355,371$

see also operations for: anus, fissures, fistula, warts

aneurisms

of common carotid artery, 398

of hand, 847

of radial artery, 588

of femoral artery, 67, 204, 222, 341, 677, 725, 785

of ophthalmic artery, 891

popliteal, 67, 204, 351, 677, 785

of temporal artery, 269

of thigh, 663

anus

artificial, 181, 794

closure of artifical, 631

closure of false, 728

see also operations for: anal

apertures

cheek, 750

roof of mouth, 517

axillary glands, 239

axillary tumour, 785,877

boil of wrist, 367

bone disease

of hand, 855

see also operations for: necrosis

breast, tumour, 19

bullet in os calcis, $881,886,891$

bullet wounds, $654,703,865$

cheek, 603

femur, 903

humerus, 680

mouth, 603

scapula, 682

see also operations for: gunshot wounds

burn contractures

axilla, near, 633

elbow, 633

hand, 291, 854

mouth, 634

burn of lip, 826

burns see operations for: cicatrices

bursae, 377

finger, 375 


\section{Index of Medical Conditions}

operations for, bursae (cont' $\mathrm{d}$ ): patellae, $257,279,422,806$

wrist, 354

cancer

cheek, $680,720,808$

ear, 887

eye, near, 732

eyelid, 624, 909

near, 789

gum, 484

hand, 711

jaw, 492

labia pudendi, 525

lip, $90,492,584,660,904,908$

lower, 494, 504, 572, 655, 666, 672, 791

upper, 523

nose, 19,726

penis, $508,559,919$

scrotum, 507,648

temple, 714

tongue, 27,597

vulva, 24

cancroid disease of eyelid, 508

cancrum oris, 306

carbuncles

abdomen, 233

back, 668

neck, $628,739,742,910$

wrist, 258

cataract, 78, 231, 356, 362, 380, 384, 390, 392, $396,404,409-410,429,451,470,478$,

$481-483,490,495,500,513,556,561,563$,

$568-570,580,581,583,586-589,621$,

$627-628,630,638,696,698,705,707-709$.

$715-716,724,734,754,760,763,869,881$

cicatrices

arm, 610

burns, 183, 208, 213, 232, 250, 393, 415, 422, 432,440

arm, 755

elbow joint, 66

throat, 788

cheek, 244

eyelid, 344

face, 836,859

and neck, 882

fingers, 468

hand, 70

knee, 808

lip, 589

neck, $488,859,882$

shoulder, 599

throat, 581

tumours, 275

recurrence of, 99,354

vagina, 208

wrist, 601

cleft palate, 498

club foot, $47,61,364,423,443,492,497$,

$566-567,572,608,610,659,663,669,690$.

$704,709,736,790,828,844,905,919$ operations for, (cont'd):

contraction of vagina, 798

corneal deposit, 661, 768

cystectomy, ovarian, 523

cysts

chest wall, 234

clavicle, near, 94

eyebrow, 333

neck, 239

ovarian, $181,198,221,223-226,368$

tapping of, 142,144

scrotum, 609

sebaceous

eye, near, 203

sacrum, 204

throat, 878

thumb, 237

deformities

eyelid, 698

legs, 257

lip, 669

lower lip, 637

nose and lip, 733

penile, 526

prepuce, 257

depressed fracture of skull, 626

dilatation of urethra, 286, 299

disease, perianal, 480

disease of

cheek, 461

elbow, 409, 411

eye, $384,388,477,760,807$

eyelid, 478,771

face, 392

femur, 606

hip, 409,426

jaw, upper, 36

knee, 834

rectum, 444

tarsus, 593

tibia, 876

toe, 409

vagina, 833

wrist, 871

dislocation of

elbow, 62, 344, 396, 644, 652, 790

femur, 867

forearm, 880

hip, $109,221,718$

humerus, $183,512,611,743,751$

shoulder, 271, 286, 361, 405, 878

old, 475

wrist, 418,430

disorder of

toe, 211

vagina, 684

distontion of femur, 488

division of

congenital fold, labia pudendi, 355 


\section{Index of Medical Conditions}

operations for, division of (cont'd): congential band of vagina, 654 lip, 724 sterno-mastoid muscle, 539, 556, 768, 826

double lip, 508

ectropion, 665, 896

elephantiasis of prepuce, 290

enchondroma

femur, 791

finger, 604

radius, 342

entropion, 455, 580, 636, 641, 710, 728, 736

epulis, 546, 574, 632, 687, 712, 717, 723, 763, 801,887

exostosis

finger, 719

humerus, 716,764

scapula, 717

tibia, 221

toe, $167,753,781$

facial pain, 26, 90, 640

fissures

anal, $267,272,285,287,377,384,387,392$, $412,484-485,549,581,592,594,632,641$, $691,710,714,727,730,736,739,803,850$, $863,890,892,907,917-918$

cheek, 63!

face, 565

lip, upper, 531

rectum, $444,483,538,581,600,628,683$, $694-695,743,797,845,859,867,872,875$

fistulae, 33, 69, 104, 220, 250, 256, 299, 323 , $342,373,387,390,404,421-422,468,540$, $546,561,657,859,887$

anal, $41,57-58,118,142,149,171,185,221$, $228,264,266,272,274,282,286-288,291$, $293,303-304,307,311,313,321-322,327$, $364-366,370,377,391,401,422,424-425$, $434,438,441-442,445-446,461,469,479$, $486,492,497,507-508,522,530,536,538$, $545,548,550,559,561-562,564,571,574$ $576-577,580-582,581,587,593-594,608$, $613,617,619,634,639-641,653,656,670$, $677,682-683,686,688,694,696,701,705$. $722,727-728,732,735,737,743,756$, $759-760,769,771,779,785,788-790,792$, $800-801,827,833,843,847-849,851,854$, $862,865,869-870,875-876,879-880,882$, $884-885,897-898,908,918$

fatal, 782-784

perianal, $241,333,351,743$

recto-vaginal, 908

recto-vesical, 277

rectum, 65]

umbilical, 560

urethral, 209

vesico-vaginal, $661,733,770,802,804,809$

foreign bodies in

airway, 673,676

bladder, 54,560 operations for, foreign bodies in (cont'd): cornea, 704, 707, 737

ear, 103,799

eye, $339,421,770$

foot, 906,917

forearm, 307

nose, 717

fractures

arm, forearm, 612

clavicle, 929

comminuted, 662

elbow, 337

femur, 751

mal-union, 337

non-union of humerus, 29

non-union of tibia, 309

tibia and fibula, 203, 872

un-united

of arm, forearm, 880,885

of femur, $150,229,919$

of humerus, 33,36

of ulna, 398

see also operations for: depressed fracture of skull

fusion of fingers, 601

ganglion, 367

gangrene of foot, 675

glands near jaw, 679

growths

conjunctiva, 673

eyelids, 87!

orbit, 871

testicle, 644

gun accident to eye, 767

gunshot wounds

abdomen, 631

from Crimea, 662

thigh, 164

see also operations for: bullet wounds

haematocele, 337, 429, 431, 484, 555

haemorrhoids, $72,78,140,158-159,169,180$, $185-186,201,210,265,267,290,299,311$, $322,327,333,336,338,355,362,368$,

$370-371,376,382,405,409,424,432,438$, $448,450,453,458,462-464,471,477-478$, $480,485-486,493,513,522,527-528,531$, $543,549-550,561,565,569,572,577-578$, $583,590,600-601,623,629,636,646,653$, $655,660,662-663,668,683,691,695$, $698-699,704,706,714,719-720,732,734$, $737,746,757,760,768,771-772,780,792$, $795,797,809,826,830-831,833,837$, $839-842,846,849,852,856,859-860$, $862-865,871-872,874,877,879,883-884$, $888,892,905,909,911,914,916$ complicated by tetanus, 892 see also haemorrhoids

hare-lip, 35, 43, 61, 72, 75, 79, 99, 105, 112, 114, $149,182-183,203,223,230,240-241$, $246-248,265,268,298,301-302,304,308$, 


\section{Index of Medical Conditions}

operations for, hare-lip (cont'd):

$326-327,336,338,346,364,374-375,385$. $389,391,393-394,397,403-404,408,413$, $415,421-422,425,427-428,441,444-445$, $450,453,455,459,464,468,475,479$, $481-482,497,499,502,507,510,523,531$, $546,554,557,559-560,567,569,572,574$, $583-585,594,596,600,609,618,621$, $624-629,637,639,647-648,658-659,663$, $669,672,696,706,714,718,723-724,727$, $733,740,745,756,762,766,772,778,785$, $787,794,809,826,835,838,858-859,867$, $869,872,887,889,895-896,899,902,905$, $908,917,919$

first stage, 871

head injury, 44

hernias, 375

incarcerated omental, 382

testis, 875

see also operations for: strangulated hernias, tumours

hydrocele, 199, 292, 900

hypospadias, 348, 376, 416, 562, 686

impacted faeces, 803

inflamed conjunctiva, 752

inflammation of eye, 916

ingrowing toe nail, 635

injury from cricket ball, $88 \mathrm{I}$

intestinal obstruction, 202

meatal stricture, 116, 231, 293, 311

melanoma

of eye, 471

of groin, 761, 863

naevi, 502, 525, 546, 555, 714, 879, 897

arm, 638

forearm, 640

axilla, 716,726

back, 392, 654, 828, 872

breast, $141,320,340,606,632,653,668$

cheek, $147,236,262,361,375,432,434,544$, 682,707

chest, $539,671,674$

chin, 750

clavicle, near, 758

eye, near, 630

eyebrow, 379

eyelid, $411,427,470,571,573,657,700,725$, 878

face, $184,270,389,714$

forehead, $229,367,376,459,535,655,681$, $723,745,803$

hand, 75

head, 200,459

jaw, 525,552

lip, 514-515, 520, 731, 828

upper, 504, 825

neck, $223,513,535,552,560,585,638,740$

nose, 361,389

occiput, 334

perianal, 62 operations for, naevi (cont'd):

scalp, 327, 393, 434, 437, 492, 501, 507, 588,

$604,618,624,633,671,692,698$

shoulder, 188,628

side, 501,585

throat, 682

trunk, 871

nasal disorders, 350

re-fashioning of new nose, $762,775,781$

septum, creation of, 567,588

tumour, 28

see also nose; operations for: polyps

necrosis of

acetabulum, 832

alveolus, 863

amputation stump, 844

ankle bone, $71,504,765,870$

bone, 403,633

of arm, 623

forearm, 614

in finger, 482,496

in foot, $257,497,703,805$

near ankle, 534

of wrist, 497

carpal and metacarpal bones, 38

carpus, $384,399,516,653,687,880$

see also wrist

clavicle, $349,589,658$

elbow, 148, 190

femur, $262,394,437,561,598,600,656,716$, $756,781,810,826,832,844,849,870,885$ head of, 202

fibula, 904

finger, 378,426

humerus, $19,53,71,115,188,210,315,344$, $453,573,601,635,641,644,661,665,696$. $701,729,825,849,914$

ilium, 434, 500, 914

jaw, 789

lower, $274,312,479,500,512,772,853,907$

upper, 188, 293, 432, 802

metacarpus, $516,683,917$

metatarsal bone, 673

metatarsus, $118,338,400,634,682,876,917$

os calcis, $37,387,401,408,499,585,734,848$, $886,891,905$

parietal bone, 437

patella, 836

pelvic bone, 919

radius, 199, 288, 593, 614, 726, 827

and ulna, 116

ribs, 153,779

sacrum, 350

scapula, 19I, 262, 769

skull, 369

sternum, $367,387,487,622,726,881$

tarsus, $385,554,585,661$

thigh, 19 


\section{Index of Medical Conditions}

operations for (cont'd):

thumb, 220, 343, 419, 500

tibia, $19,21,32,37,47,53,68,89,112,118$, $229,261-262,280,288,300,316,338,368$, $370-371,375,378,387,391,393,395,398$, $400,407-409,417,425,444,451,458,464$, $503-504,515,520,526,562,573,585,624$, $661,699,709,711,718,725,730,736,755$, $765,791,811,841,844-845,848,861,863$, 879,915

toe, $38,262,629$

ulna, $116,355,385,573,726$

wrist, $418,464,562,699,887$

see also carpus

neuralgia

arm, 574

face, 219

see also neuralgia

osteomyelitis of tibia, 421

penile

scarring, 423

tags, 292

see also operations for: adhesions, deformities, warts; penis; prepuce

perianal

tags, 63

see also operations for: abscesses, fistulae, naevi, sinuses

perineal see operations for: abscesses, disease, rupture, sinuses

phymosis, 150, 162, 167, 208, 220, 223, 248, 254, $283,325,333,340,357,368,382,385,407$, $416,420,423,429,437,444,491,498,588$, $597,621,701,707,710-711,713,718,737$, $833,847,896,908,910$

plastic, nose and lip, 697

polyps

anal, 479

aural, $274,366,379,652$

nasal, $55,78,256,259,476,497,520,678,686$, 707,904

post-operative incompetence of anus, 637

procidentia uteri, 805,873

prolapse

anal, $8,35,126,294,299,311,313,338,341$, $382,412,446,462,474,477-478,484,534$, $536,540,554,579,600,635,673,691,699$, $706,720,771,803,809,825,832,837$, $841-842,846,860,879,892,914$

complicated by tetanus, 892

rectum, 427

uterus, $791,836,849,854,885$

vaginal, 530

protrusion of iris, 712

pterygium, 366

pupil, artificial, $406,412,552,555,754,788$, $862,864,906$

rupture of perineum, $207,318,324,370,398$, $530,633,690,775,779,807,840,888,892,911$ scrofulous testicle, 13 operations for (cont'd):

scrotal warts, 208

sequestrum in bladder, 192

sinuses, 645,705

amputation stump, 502

ankle, near, 525

breast, 153

buttocks, 850

cheek, 836

face, 863

groin, 729

ilium, 417

near, 524

jaw, from, 836

knee, 887

near, 574,732

leg, 743

nose, bridge of, 519

perianal, $84,141,313,345-346,453,469,561$, $645,668-669,699,731$

perineal, $32,38,89,116,232,480,592,619$, 623,671

rectum, 148,710

ribs

between, 845

near, 351

sacrum, over, 244,580

scapula, 244

scrotum, 730

thigh, 865

urethra, 198, 703

sloughing of lower lip, 708

staphyloma, 772, 873

stone

prostatic, 576

urethral, 576

see also bladder; lithotomy; lithotrity

strabismus, 306, 318, 327, 331, 333-334, 365,

$391,397,430,439,477,486,496,500,540$,

$543,548,622,628,648,661,677,683,691$,

$707,715,753-754,759-760,762,764$,

$777-778,781,793,797-798,800,802,808$,

$830-831,840,842,845,849,858,889,895$,

$898-902,904,907$

post-operative, 901

strangulated hernias, $162,200,212,273,348$, $369,550,679,746,830$

femoral, 334, 554, 688

inguinal, 511,625

umbilical, 402, 570, 889

see also operations for: hernias

strictures, 547,736

rectal, 353, 502, 540, 732, 779

Syme's operation for, 736

urethral, $60,104,119,121,132,156,170,177$ $183,192,211,213,218,238,267,269,280$, $289,292,308,324,326-327,337,352,385$, $405,418,438,440,442,448,452-453,457$, $463,503-504,550,556,581,587,600,607$, $646,656,672,675,678,681,704,708,731$, $758,763,766,795,844,856,885$ 


\section{Index of Medical Conditions}

operations for (cont'd):

swelling involving orbit, 557

syndactyly, 119, 412

syphilis of nose, 912

syphilitic bone disease, 598

trauma to fingers, 547

traumatised eye, 681

tumours

abdomen, 432

anus, 276,349

arm, 19, 345, 379, 440,649, 706

axilla, $73,192,210,236,370,373,440$,

$460-461,568,590,610,656,681,686,785$, $867-868,877$

back, 27, 43, 54, 103, 110, 258, 321, 337, 411, $431,579,582,664-665,667$

buttock, 703

cervix, 35

cheek, 211, 221, 235, 264, 374, 377, 485, 505,

$532,539,544,564,574,697$

chest wall, 207, 281, 394

chin, near, 753

clavicle, near, 97,555

condylomata of labia pudendi, 113

ear, 19.63

behind, 584

elbow, 312

near, 700

epididymis, 719

eye, 95,522

near, $35,641,677$

eyebrow, 62, 214, 234, 290, 394, 597, 643, $747-748,804,806,848$

eyelid, 43, 87, 312, 337, 372, 424, 456, $460-461,480,500,545,583,708,733,809$, 854,867

face, $81,84,122,160,189,317,373,496,512$, $527,570,573,597,873$

femur, 120

finger, 599

flank, 238, 267

foot, $25,258,737$

vascular, 258

forehead, 31, 209, 212, 310, 558, 701, 776, 805, 863

gastrocnemius muscle, beneath, 516

groin, 111, 145, 207, 254, 476, 848-849

melanotic, 248, 260, 365

hand, $148,371,378$

head, 116

hernial, 510

hip, 488

ilium, 176, 837

near, 665,692

jaw, 137, 357, 374, 377, 383, 419, 619, 706, 735,794

angle of, 146, 538

below, 310

lower, 28, 31, 244, 459-460, 546, 580, 621, $798,828,912$ operation for, tumours, jaw, lower (cont'd): near, 706

near, $675,681,692,694,719,723,744,831$, 861,904

upper, $36,145,155,317,355,389,664,697$, 714,832

knee, 364

labia pudendi, $83,91,96,119,206,555,595$. 651,705

larynx, 617

leg, $74,327,453,704$

lip, 47, 120,181, 269, 325, 364, 442, 64 !

lower, 487

loin, 402

malar bone, 165

maxillary antrum, 210,417

meatus urinarium, 508

median nerve, 902

mouth, 233, 785

nates, $203,379,569,718-719$

neck, 12, 31, 217, 224, 259, 307, 327, 388, 391, $401,424,436-437,443,464,518,555,569$, $627,660,685,711,770,801,868,877,891$, 900

nose, $260,411,449,655,686,708,851$

bridge of, 519

nostril, 395

orbit, $60,118,305,617,634,653,680$

near, 33,558

ovarian, 332, 761

parotid gland, over or near, 147, 159, 513,666,

706,709

penis, $235,262,322,329,443,456$

perineum, 383

pinna, 268

posterior tibial nerve, 630

pubes, 608

rectum, 303,503

sacrum, 68,216

near, 685

scalp, 96, 266, 280, 518, 621, 627, 696, 716,

835,891

sebaceous, 9

scapulae, 6, 24

between, $113,718,899-900$

over, 528

see also shoulders

scrobiculus cordis, 77

scrotum, 316, 393, 696, 738

shoulders, 26, 69, 81-82, 85-86, 105, 160, 319 , $329-330,352,359,364,402,417,507,530$,

$583,590,624,674,677,692,714,794$

between, 219,792

near, $279,683,733$

see also scapulae

side, 337,520

skull, 305

sterno-mastoid muscle, near, 839

sternum, 164, 376

temple, $541,792,797$ 


\section{Index of Medical Conditions}

operations for, tumours (cont'd):

testicle, 33, 39, 726, 898

thigh, $42,53,77,148,354,363,376,391,463$, $487,598,701,717,729,763,785,909$

thumb, 452

thyroid, 614

thyroid cartilage, near, 781

tibia, $665,846,851,899$

tongue, $95,187,279,283,358$

umbilicus, 119

urethra, 406,685

uterus, 280

vagina, $122,260,315,346,349-350,451,500$, 901

vascular, of foot, 258

wrist, 342,487

see also breast tumours; operations for:

cicatrices, enchondroma

urethral

diverticulum, 283

stone, 153,636

see also operations for: sinuses, strictures,

fistulae, tumours

urethrostomy, 712

varicocoele, 875

varicose veins, 448,851

warts

anal, $234,246,331,628$

labia pudendi, 135, 146, 266, 299, 303-304, 313,344

penile, $6,24,220,311,397,415,575-576,603$,

790,888

scrotal, 208

vaginal, 438

vulval, 288-289, 297, 912

webbed fingers, $776,780,804$

whitlow, 765

thumb, 647

ophthalmia

purulent, 694

sympathetic, 731

opiates, 538

see also opium

opisthotonos, $552,752,770,777,780$

during induction, 503, 521

opium

administration, 529

tincture of, 868

orbit see eyes

orchidectomy, $13,53,79,134-135,248-249,256$,

$266,294,309,346,379,425,445,534,539,618$,

$710,792,795$

os calcis see operations for: necrosis, bullet

osteomyelitis of tibia, 317,421

osteosarcoma of shoulder, 359

ovarian tumour, with debility, co-existent disease, 761

ovaries see operations for: cystectomy, cysts
$\mathrm{P}$

pain

back, chloroform administered for, 283

chloroform administered for, 46, 63, 195-196

post-operative, chloroform administered for, 92 ,

$147,180,281$

see also facial pain

Palorcotian operation, 707

palpitations, co-existent disease, 65

paracentesis abdominis, 101, 163

paracentesis thoracis, 174,178

parietal bone, necrosis of, 437

parotid gland, tumours over or near, $147,159,513$, $666,706,709$

patella, necrosis of, 836

pelvic abscess, 828

pelvic bone, necrosis of, 919

penis

cancer, 508, 559, 919

see also amputations; circumcision; operations

for: penile, tumours; prepuce

perineum

tearing of, 87

tumour, 383

see also operations for: abscesses, rupture of

perineum, sinuses

peritonitis

death from, 631

pelvic, 503

phthisis, co-existent disease, 530,534

phymosis see operations for: phymosis

pinna, tumour, 268

Pirogoff's amputation, 891-892, 897

placenta

manual removal of, 647,834

retained, 253,445

plantar, tenotomy, 356

pleuro-pneumonia, fatal, 560

polycythaemia, co-existent disease, 52

polyps see operations for: polyps

post-operative

analgesia, chloroform administered for, 47,78 , 732

bradycardia, 513

complication, immediate, 471,474

debility, 479

hysteria, 472, 484, 496, 511

incompetence of anus, 637

pleuro-pneumonia, fatal, 560

respiratory obstruction, 492

shivering, 477

singing, 49

stimulants

ammonia, 243, 319, 511

brandy, 852,884

sherry, 866

post-partum neuralgia, 499

pregnancy

anaesthesia during, $6,290,323,369,381,468$, $472,493,608,709,807,858,865,885$ 


\section{Index of Medical Conditions}

pregnancy (cont'd):

see also depression

prepuce

adhesion, 689

deformity, 257

elephantiasis, 290

nitric acid applied, 514, 629

see also circumcision; penis

presentation

arm, 805

breech, 650

shoulder, 476

prolapse, see also operations for: prolapse

prostate, enlargement of, 514

prostatic calculi, 308, 576, 636

pterygium, 366

pubes, tumour, 608

puerperal insanity, 510

puerperium, anaesthesia during, 475

pullies, use of, $183,221,271,344,406,475$

pulselessness due to an artifact, 522

pupils see eyes

purpura haemorrhagica, 934

$\mathbf{R}$

radial pulse, anatomical absence of, 800

radius, necrosis of, $199,288,593,614,726,827$

recti, divarication of, 722

rectum

abscess, 651

disease, 444

examinations, $443,679,734-735,757,900$

for malignant disease, 653

fissure, nitric acid applied, 650

intra-operative laceration of, 721

prolapse, 427

sinuses, 148,710

strictures, $353,502,540,732,779$

tumours, 303, 503

ulcer, 11

see also operations for: fissures

renal disease, co-existent disease, 903

respiratory disorder, Snow manages a, 170-180

resuscitation

neonatal, 805

Marshall Hall's method, 796

mouth to mouth, 650,702

post-operative, 472

rheumatic heart disease, co-existent disease, 474

rheumatism, 2, 4, 7

ribs

necrosis of, 153,779

sinuses

between, 845

near, 351

rickets, co-existent disease, 471

rigor, 4-5

road accident, 3

Royal Oak Benefit Society, examination of patient at, 938
$S$

sacrum

cyst, sebaceous, 204

necrosis of, 350

sinuses over, 244,580

tumours, 68,216

near, 685

Salmon, Mr.

dissatisfied with operating conditions, 619

has poor operating conditions, 546

operates before Snow is ready, 550

scalp

tumour, sebaceous, 9

see also operations for: naevi, tumours

scapula

bullet wound, 682

deformity of, 774

exostosis, 717

necrosis of, 191, 262, 769

sinus, 244

see also operations for: tumours

scarlet fever, 154, 708

scars

recurrence of tumour in, 99, 320, 354

see also operations for: cicatrices

sciatic nerve, neuroma of, 468

sclerosant

injection of orbit with, 725

see also tannic acid

scrobiculus cordis, tumours, 77

scrotum

cancer, 507,648

cyst, 609

sinuses, 730

tumours, 316, 393, 696, 738

warts, 208

see also testicles

seton

insertion of, to an un-united fracture, 662

introduction of, 878

sherry, as a post-operative stimulant, 866

shivering, post-operative, $71,84,395$

shoulder

abscess near, 625

bandaging of, 319

cicatrix, 599

manipulation of, $780,799,827$

naevi, 188,628

osteosarcoma, 359

see also operations for: dislocation, tumours

shoulder blade see scapula

side

naevi, 501,585

tumours, 337, 520

see also flank

silver nitrate

application of, 695

eye, 694

face, 935

naevus, 803

os uteri, 884 


\section{Index of Medical Conditions}

singing

during operation, by patient, 844

post-operative, 548

skin grafting, 599, 896

skull

depressed fracture of, 626

necrosis of, 369

tumours, 305

see also head; parietal bone

Snow

consults about advisability of using chloroform, 586

examines chest of patient before operation, 534

leaves before delivery of second twin, 603

leaves before labour completed, 646, 800, 806

leaves a pulseless patient, 529

uses contaminated chloroform, 593

sofa, operating, moved during anaesthesia, 565

speculum, examination with, under chloroform, 635

sphincterotomy, 803

anal, 11, 134, 862

spinal disorder, co-existent disease, 900

sponge

amylene on a, $740,745,775,811$

chloroform on a, 492, 750, 763, 802

via laryngotomy, 660, 673, 676

chloroform and spirit on a, 485, 580, 621, 851

ether on a, 756, 762

used as well as inhaler, via laryngotomy, 660

staphyloma, 697, 772, 873

steatoma, 723

sterno-mastoid muscle

division of, $564,568,836,842,861$

tenotomy, 188

tumour near, 839

sternum

necrosis, $367,387,487,622,726,88$ I

tumours, 164, 376

still-births, 250,360

stitches, insertion of, after anaesthesia, 498

stone see operations for: stone

strabismus see operations for: strabismus

strangury, 3

strictures see operations for: strictures

struggling

during induction, $528,531,544,561,565$,

$575-576,590,605,612,614,628,645,719$,

$751-752,770,825,874,887,899$

intra-operative, $482,487,494$

suffocation, incipient, during tonsillectomy, 537

suicide, attempted, during pregnancy, 509

surgery, prone position for, 35

Syme's amputation, 618

Syme's operation for stricture, 736

syndactyly, 119,412

syphilis, 268

see also operations for: syphilis

syringe, female, 14

syringing of ears, 164
$\mathrm{T}$

tags

anal, $1,82,231,237,307,309,350,355,371$

penile, 292

perianal, 63

tannic acid as a sclerosant, 725

tarsus

disease of, 593

necrosis of, $385,554,585,661$

taxis for hernia, 510

temple

cancer, 714

tumours, $541,792,797$

temporal artery thickening, 646

tenesmus, 3

tenotomy, 242, 566-567, 811

Achilles, 61, 75, 110, 172, 188, 192, 231, 257,

$288,310,320,356,599,693,791,799,827$,

$835,843,906$

adductor, 867

ankle, 219,915

biceps, 750

fingers, 738

foot, $47,110,150,156,219,364,367,443,694$,

$733,736-737,765,768,774,777,780$,

$798-799,803-804,807,811,827,871,891$

ham-string, $2,24,419,458,470,659,796,808$, $832,871,887,889,891,914$

hand, 460

knee, near, 745

plantar, 356

post-amputation, 230

rectus femoris, 840

sterno-mastoid muscle, 188

thigh muscles, 230

thumb, 777

toes, 799

triceps, 864,880

wrist, $693,545,661$

testicles

growth on, 644

maldescended, 523

scrofulous, 13

tumours, 33, 39, 726, 898

see also hydrocele; orchidectomy; scrotum

tetanus

chloroform administration for, $64-65,506,892$

co-existent disease, 64-65

deaths from, 506, 894

thigh

abscess, $164,399,514,668,685$

aneurism of, 663

manipulation of, 591

necrosis of, 19

sinuses, 865

see also amputations; femur; legs; operations for:

throat

tumours; tenotomy

burn cicatrix, 788

cicatrices, 581 


\section{Index of Medical Conditions}

throat (cont'd):

cyst, sebaceous, 878

naevus, 682

see also larynx; neck

thumb

abscess, 392

cyst, operation for, 237

necrosis of, $220,343,419,500$

neurectomy, 389

nitric acid applied, 765

tenotomy, 777

tumour, 452

whitlow, 647

see also amputations; fingers

thyroid, tumour, 614

thyroid cartilage, tumour near, 781

tibia

disease of, 876

excision of, 218

exostosis, 221

fracture, 203,872

non-union, 309

node, 78

osteomyelitis, 317,421

resection of, 218

tumours, $665,846,851,899$

see also operations for: necrosis; trephining

tic, sulphuric ether inhalation for a, 787

toes

disorder of, 211

examination of toe bone, 643

exostosis, $167,753,781$

ingrowing nail, 635

necrosis of, $38,262,629$

operation for abscess, 767

tenotomy, 799

see also amputations; nails, toe-

Tomes dilator, use of, 473

tongue

cancer, 27,597

tumours, 95, 187, 279, 283, 358

tonsillectomy, 537, 651, 831, 892

tooth

narcotic application to, 666

nitric acid applied, 578

tourniquet, faulty, leads to haemorrhage, 829

towel, chloroform on a, 853

tracheal tube, insertion of, 726

tracheostomy, 328

trephining

of femur, 604

of tibia, 317, 378, 395, 411, 446, 602, 860, 867, 892,900

triceps, tenotomy, 864,880

trunk, naevus on, 871

tuberculosis

co-existent disease, 706,878

see also phthisis

tumours

kidney, 654

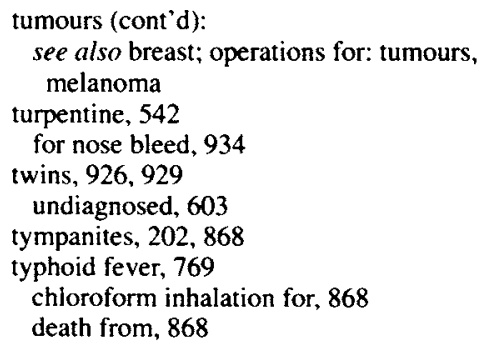

\section{$\mathrm{U}$}

ulcer, rectal, II

ulna, necrosis of, $116,355,385,573,726$

umbilical fistula, 560

umbilicus, tumour, 119

un-named operation, 19

urethra

dilatation of, 286, 299

intra-operative trauma to, 590

rupture of, 192

tumour, 406

see also operations for: strictures, urethrostomy urethrotome, 213,218

urine

extravasation of, $601,607,638$

retention of, 301

see also albuminuria; anuria

uterus

procidentia uteri, 805,873

prolapsed, $791,836,849,854,885$

tumour, 280

$\mathrm{V}$

vaccination, $14,21,24,27,62,96,109,113,189$ $210,242,320,354,383,454,458,927$. $929-931$

on nose, 458

vagina

contraction of, 798

disease of, 833

disorder of, 684

division of congenital band, 654

malformed, examination of, 799

see also operations for: adhesions, tumours, warts valves

closed during anaesthesia and inhaler removed, 510

closure

inadvertent causes death, 782-784

Snow afraid to complete, 781

kept open during hyperventilation, 496

not closed, patient lightens, 611

not so much closed as usual, 536

only half closed, 638

problem with the expiratory, 489

very much open, 672

see also inhaler 


\section{Index of Medical Conditions}

veins, spermatic, ligation of, 771 version

cephalic, 138

of child, intra-partum, 476

during labour, 602, 805

vitreous humour, escape of, 709

vomiting, intra-operative, 491

vulva, cancer, operation for, 24

\section{W}

warts see operations for: warts

wen, 697

Whitlow, 294, 304, 307, 350, 405, 505, 647, 765

whooping cough see hooping cough wrist

boil, 367

bursa, 354

carbuncle, 258

cicatrix, 601

disease of, 871

dislocation of, 418,430

necrosis of, 384, 399, 418, 464, 497, 516, 562,

$653,687,699,880,887$

tenotomy, $545,661,693$

tumours, 342,487

see also amputations; carpal bones; operations for: necrosis of carpus

wry neck, 188 